\title{
Some new inequalities for continuous fusion frames and fusion pairs
}

\author{
Wei Zhang ${ }^{*}$ and Yun-Zhang Li
}

*Correspondence: zwfylhappy@126.com College of Applied

Sciences, Beijing University of Technology, Beijing 100124, People's Republic of China

\begin{abstract}
This paper addresses continuous fusion frames and fusion pairs which are extensions of discrete fusion frames and continuous frames. The study of equalities and inequalities for various frames has seen great achievements. In this paper, using operator methods we establish some new inequalities for continuous fusion frames and fusion pairs. Our results extend and improve ones obtained by Balan, Casazza and Găvruţa.
\end{abstract}

Keywords: Continuous fusion frame, Parseval continuous fusion frame, Fusion pair Mathematics Subject Classification (2010): 42C15, 42 C 40

\section{Background}

The notion of frame in a general Hilbert space was first introduced by Duffin and Schaeffer in 1952 to study nonharmonic Fourier series (Duffin and Schaeffer 1952). However, the frame theory had not interested many researchers until Daubechies, Crossman and Meyer published their ground breaking work in Daubechies et al. (1986). In recent years, the study of frame theory has seen great achievements, and frames are widely used in signal processing, quantum measurements, image processing, coding and communication and some other fields (Balan et al. 2007; Bownik et al. 2015; Casazza 2000; Christensen 2003; Leng and Han 2013; Li and Sun 2008; Li and Zhu 2012; Li et al. 2015; Rahimi et al. 2006; Strohmer and Heath 2003). The study of fusion frames dates back to Casazza and Kutyniok (2004) by Casazza et al. (2008) by Casazza, Kutyniok and Li, which can be used in distributed processing. As Casazza, Kutyniok and Li pointed out in Casazza et al. (2008), in applications, one is often overwhelmed by a deluge of data assigned to one single frame system, which becomes simply too large to be handled numerically. In these cases it would be highly beneficial to split a large frame system into a set of (overlapping) much smaller systems, and to process locally within each subsystem effectively. The notion of continuous fusion frame is a generalization of the above discrete fusion frames, It was first introduced by Faroughi and Ahmadi in Faroughi and Ahmadi (2008). This paper focuses on some inequalities for continuous fusion frames and fusion pairs.

In applications such as speech recognition, it was a longstanding conjecture by many engineers that a signal can be reconstructed without information about the phase. In 2006, Balan, Casazza and Edidin verified this conjecture (Balan et al. 2006). While

(c) 2016 The Author(s). This article is distributed under the terms of the Creative Commons Attribution 4.0 International License (http://creativecommons.org/licenses/by/4.0/), which permits unrestricted use, distribution, and reproduction in any medium, provided you give appropriate credit to the original author(s) and the source, provide a link to the Creative Commons license, and indicate if changes were made. 
working on efficient algorithms for signal reconstruction, Balan, Casazza, Edidin and Kutyniok in Balan et al. (2005) pointed out the following surprising proposition, and proved it detailedly in Balan et al. (2007).

Proposition 1 (Balan et al. 2005, Theorem 3.2) Let $\left\{f_{i}\right\}_{i \in I}$ be a Parseval frame for $\mathcal{H}$. For every subset $J \subset I$ and every $f \in \mathcal{H}$, we have

$$
\sum_{i \in J}\left|\left\langle f, f_{i}\right\rangle\right|^{2}-\left\|\sum_{i \in J}\left\langle f, f_{i}\right\rangle f_{i}\right\|^{2}=\sum_{i \in J^{c}}\left|\left\langle f, f_{i}\right\rangle\right|^{2}-\left\|\sum_{i \in J^{c}}\left\langle f, f_{i}\right\rangle f_{i}\right\|^{2} .
$$

Then the study of inequalities related to (1) has interested many mathematicians. The details can be found in Găvruţa (2006); Guo et al. (2016); Li and Sun (2008); Li and Zhu (2012); Zhu and Wu (2010) and references therein. In particular, Balan, Casazza, Edidin and Kutyniok in 2007 and Găvruţa in 2006 obtained following two propositions:

Proposition 2 (Balan et al. 2007, Proposition 4.1) Let $\left\{f_{j}\right\}_{j \in J} \subset H$ be a Parseval frame. For any $f \in H, J_{1} \subset J$, we have

$$
\sum_{j \in J_{1}}\left|\left\langle f, f_{j}\right\rangle\right|^{2}+\left\|\sum_{j \in J_{1}^{c}}\left\langle f, f_{j}\right\rangle f_{j}\right\|^{2} \geq \frac{3}{4}\|f\|^{2} .
$$

Proposition 3 (Găvruţa 2006, Theorem 3.2) Let $\left\{f_{j}\right\}_{j \in J} \subset H$ be a frame and $\left\{g_{j}\right\}_{j \in J} \subset H$ be an alternate dual frame of $\left\{f_{j}\right\}_{j \in J}$. then for any $f \in H$, we have

$$
\operatorname{Re}\left(\sum_{j \in J_{1}}\left\langle f, g_{j}\right\rangle \overline{\left\langle f, f_{j}\right\rangle}\right)+\left\|\sum_{j \in J_{1}^{c}}\left\langle f, g_{j}\right\rangle f_{j}\right\|^{2} \geq \frac{3}{4}\|f\|^{2} .
$$

Guo, Leng and Li in Guo et al. (2016) generalized Proposition 1 to discrete fusion frames (Guo et al. 2016, Theorem 4). Motivated by above works, in this paper we generalize Proposition 2 and Proposition 3 to continuous fusion frames and fusion pairs. It is worth expecting that our results have potential applications in the frame theory and signal processing. Indeed, our results can be used to recover many results in the literature. For example, Theorem 1 below reduces to Guo et al. (2016), Theorem 8) when the measure is counting measure, and to Proposition 2 if the fusion frame is taken as the usual frame in addition. Similarly, Corollary 3 can be used to recover Proposition 3.

This paper is organized as follows. "Preliminaries" section is an auxiliary one. And in this section, we recall some basic notions and properties. In "Equalities and inequalities for continuous fusion frames" section, using the method of operator theory we obtain some important inequalities for continuous fusion frames which are very different from those in the literature. In "Equalities and inequalities for fusion pairs" section, we derive some inequalities of fusion pairs and some bounds estimates. 


\section{Preliminaries}

This section is an auxiliary one. First we recall some basic notations and notions . The readers can refer to Casazza and Kutyniok (2004), Christensen (2003), Faroughi and Ahmadi (2008, 2010), Rahimi et al. (2006) for details.

Let $H, K$ be separable Hilbert spaces, and $I$ a countable index set. We denote by $I_{H}$ the identity operator on $H, \hat{H}$ the collection of all closed subspace of $H$, and $L(H, K)$ the set of all bounded linear operators from $H$ into $K$. For a positive measure space $(X, \mu)$, we always assume that $v: X \rightarrow[0, \infty)$ is measurable mapping on $X$ satisfying $v(x) \neq 0$ for a.e. $x \in X$.

Let $F$ be a mapping from $X$ into $\widehat{H}$. We denote by $L^{2}(X, F)$ the set of all measurable mappings $f: X \rightarrow H$ such that, for each $x \in X$, and $f(x) \in F(x)$, and $\int_{X}\|f(x)\|^{2} d \mu(x)<\infty$. Then it is a Hilbert space under the following inner product:

$$
\langle f, g\rangle=\int_{X}\langle f(x), g(x)\rangle d \mu(x) \quad f, g \in L^{2}(X, F) .
$$

Definition 1 (Christensen 2003) Let $\left\{f_{i}: i \in I\right\}$ be a sequence in $H$, we say that $\left\{f_{i}: i \in I\right\}$ is a frame if there exist $0<A_{1} \leq B_{1}<+\infty$ such that

$$
A_{1}\|h\|^{2} \leq \sum_{i \in I}\left|\left\langle f_{i}, h\right\rangle\right|^{2} \leq B_{1}\|h\|^{2}, \quad \forall h \in H .
$$

The numbers $A_{1}, B_{1}$ are called lower and upper bounds for the frame, respectively.

Definition 2 (Casazza and Kutyniok 2004) Let $H$ be a separable Hilbert space, $\left\{w_{i}: i \in I\right\}$ be a family of closed subspace of Hilbert space $H$, and $\left\{v_{i}: i \in I\right\}$ be a family of weight, i.e., $v_{i}>0$ for all $i \in I$. The family $\left\{\left(w_{i}, v_{i}\right): i \in I\right\}$ is a fusion frame, if there exist constants $0<A_{2} \leq B_{2}<+\infty$ such that

$$
A_{2}\|h\|^{2} \leq \sum_{i \in I} v_{i}^{2}\left\|\pi_{w_{i}}(h)\right\|^{2} \leq B_{2}\|h\|^{2}, \quad \forall h \in H,
$$

where $\pi_{w_{i}}$ is the orthogonal projection onto the subspace $w_{i}$. The numbers $A_{2}, B_{2}$ are called lower and upper frame bounds for the fusion frame, respectively.

Definition 3 (Rahimi et al. 2006) Let $(X, \mu)$ be a measure space with positive measure $\mu$, and let $f: X \rightarrow H$ be weakly measurable (i.e., for all $h \in H$, the mapping $x \rightarrow\langle f(x), h\rangle$ is measurable). Then $\{f(x): x \in X\}$ is called a continuous frame for $H$ if there exist constants $0<A_{3} \leq B_{3}<+\infty$ such that

$$
A_{3}\|h\|^{2} \leq \int_{X}|\langle f(x), h\rangle|^{2} d \mu(x) \leq B_{3}\|h\|^{2}, \quad \forall h \in H .
$$

We call $A_{3}$ and $B_{3}$ the lower and upper continuous frame bounds, respectively. If only the right-hand inequality of (4) is satisfied, we call $\{f(x): x \in X\}$ a continuous Bessel sequence in $H$ with Bessel bound $B_{3}$. If $A_{3}=B_{3}=\lambda$ in (4), we call $\{f(x): x \in X\}$ a $\lambda$-tight continuous frame. Moreover, if $\lambda=1,\{f(x): x \in X\}$ is called a Parseval continuous frame. 
Definition 4 (Faroughi and Ahmadi 2008) Let $F: X \rightarrow \widehat{H}$ be such that for each $h \in H$, the mapping $x \rightarrow \pi_{F_{(x)}}(h)$ is measurable (i.e., $F$ is weakly measurable). We say that $(F, v)$ is a continuous fusion frame for $H$ if there exist constants $0<A \leq B<+\infty$ such that

$$
A\|h\|^{2} \leq \int_{X} v^{2}(x)\left\|\pi_{F(x)}(h)\right\|^{2} d \mu(x) \leq B\|h\|^{2}, \quad \forall h \in H,
$$

where $\pi_{F(x)}$ is the orthogonal projection onto the space $F(x)$. The numbers $A, B$ are called lower and upper frame bounds for the continuous fusion frame, respectively. If only the right-hand inequality of (5) is satisfied, we call $(F, v)$ a continuous Bessel fusion mapping on $H$ with bound $B$. If $A=B=\lambda$ in (5), we call $(F, v)$ a $\lambda$-tight continuous fusion frame. Moreover, if $\lambda=1,(F, v)$ is called a Parsevel continuous fusion frame.

Remark 1 A continuous fusion frame is a generalization of fusion frame. Indeed, when $X$ is countable, and $\mu$ is a counting measure, it is exactly a fusion frame.

Let $(F, v)$ be a continuous fusion frame for $H$. In Faroughi and Ahmadi (2008), the authors defined the continuous fusion frame operator $S_{F}: H \rightarrow H$ as follows:

$$
S_{F}(h)=\int_{X} v^{2}(x) \pi_{F(x)}(h) d \mu(x), \quad \forall h \in H .
$$

It is easy to show that $S_{F}$ is a bounded, positive, self-adjoint and invertible operator.

For any $X_{1} \subset X$, denote $X_{1}^{c}=X \backslash X_{1}$, and we define the following operators:

$$
\begin{aligned}
& S_{F}^{X_{1}} h=\int_{X_{1}} v^{2}(x) \pi_{F(x)}(h) d \mu(x), \quad \forall h \in H . \\
& S_{F}^{X_{1}^{c}} h=\int_{X_{1}^{c}} v^{2}(x) \pi_{F(x)}(h) d \mu(x), \quad \forall h \in H .
\end{aligned}
$$

Then $S_{F}=S_{F}^{X_{1}}+S_{F}^{X_{1}^{c}}$, and $S_{F}^{X_{1}}, S_{F}^{X_{1}^{c}}$ are positive and self-adjoin operators.

Definition 5 (Faroughi and Ahmadi 2010) Let $(F, v)$ and $(G, v)$ be continuous Bessel fusion mappings on $H$. We say that $F$ and $G$ is a fusion pair if for any $h \in H$ the following holds

$$
h=\int_{X} v^{2}(x) \pi_{G(x)} \pi_{F(x)}(h) d \mu(x)=\int_{X} v^{2}(x) \pi_{F(x)} \pi_{G(x)}(h) d \mu(x) .
$$

\section{Equalities and inequalities for continuous fusion frames}

This section is devoted to some inequalities for continuous fusion frames. For this purpose, we first give a simple property of self-adjoint operators.

Lemma 1 Let $T \in L(H)$ bea self-adjointoperatorand $a, b, c \in \mathcal{R}, U=a T^{2}+b T+c I_{H}$, then the following statements hold. 
(i) if $a>0$, then

$$
U \geq \frac{4 a c-b^{2}}{4 a} I_{H}
$$

(ii) if $a<0$, then

$$
U \leq \frac{4 a c-b^{2}}{4 a} I_{H}
$$

Proof We only prove (i), and (ii) can be proved similarly. It is easy to check that

$$
U=a\left(T+\frac{b}{2 a} I_{H}\right)^{2}+\frac{4 a c-b^{2}}{4 a} I_{H}
$$

Observing that $\left(T+\frac{b}{2 a} I_{H}\right)^{2}$ is a positive operator, we have (i).

Lemma 2 (Găvruţa 2006, Theorem 2.1) If $T_{1}, T_{2} \in L(H)$ are bounded, self-adjoint linear operator satisfying $T_{1}+T_{2}=I_{H}$, then for all $h \in H$, we have

$$
\left\langle T_{1} h, h\right\rangle+\left\|T_{2} h\right\|^{2}=\left\langle T_{2} h, h\right\rangle+\left\|T_{1} h\right\|^{2} \geq \frac{3}{4}\|h\|^{2} .
$$

Theorem 1 Let $(F, v)$ be a Parseval continuous fusion frame for $H$. Then for $X_{1} \subset X$ and $h \in H$, we have

$$
\begin{aligned}
& 0 \leq \int_{X_{1}} v^{2}(x)\left\|\pi_{F(x)}(h)\right\|^{2} d \mu(x)-\left\|\int_{X_{1}} v^{2}(x) \pi_{F(x)}(h) d \mu(x)\right\|^{2} \leq \frac{1}{4}\|h\|^{2}, \\
& \frac{1}{2}\|h\|^{2} \leq\left\|\int_{X_{1}} v^{2}(x) \pi_{F(x)}(h) d \mu(x)\right\|^{2}+\left\|\int_{X_{1}^{c}} v^{2}(x) \pi_{F(x)}(h) d \mu(x)\right\|^{2} \leq \frac{3}{2}\|h\|^{2}, \\
& \frac{3}{4}\|h\|^{2} \leq \int_{X_{1}} v^{2}(x)\left\|\pi_{F(x)}(h)\right\|^{2} d \mu(x)+\left\|\int_{X_{1}^{c}} v^{2}(x) \pi_{F(x)}(h) d \mu(x)\right\|^{2} \leq\|h\|^{2} .
\end{aligned}
$$

Proof Suppose that $(F, v)$ is a Parseval continuous fusion frame for $H$, then $S_{F}$ is invertible and positive on $H$ and $S_{F}^{X_{1}}+S_{F}^{X_{1}^{c}}=I_{H}$. By a simple calculation, we have $S_{F}^{X_{1}} S_{F}^{X_{1}^{c}}=S_{F}^{X_{1}^{c}} S_{F}^{X_{1}}$. It follows that

$$
0 \leq S_{F}^{X_{1}} S_{F}^{X_{1}^{c}}=S_{F}^{X_{1}}\left(I_{H}-S_{F}^{X_{1}}\right)=S_{F}^{X_{1}}-\left(S_{F}^{X_{1}}\right)^{2},
$$

and thus

$$
S_{F}^{X_{1}}-\left(S_{F}^{X_{1}}\right)^{2} \leq \frac{1}{4} I_{H}
$$


by Lemma 1 . For $h \in H$, we have

$$
\begin{aligned}
\left\langle\left(S_{F}^{X_{1}}-\left(S_{F}^{X_{1}}\right)^{2}\right) h, h\right\rangle & =\left\langle S_{F}^{X_{1}} h, h\right\rangle-\left\langle\left(S_{F}^{X_{1}}\right)^{2} h, h\right\rangle \\
& =\int_{X_{1}} v^{2}(x)\left\|\pi_{F(x)}(h)\right\|^{2} d \mu(x)-\left\|\int_{X_{1}} v^{2}(x) \pi_{F(x)}(h) d \mu(x)\right\|^{2} .
\end{aligned}
$$

Combining (9) and (10), we get (6).

Observe that

$$
\begin{aligned}
\left(S_{F}^{X_{1}}\right)^{2}+\left(S_{F}^{X_{1}^{c}}\right)^{2} & =\left(S_{F}^{X_{1}}\right)^{2}+\left(I_{H}-S_{F}^{X_{1}}\right)^{2} \\
& =2\left(S_{F}^{X_{1}}\right)^{2}-2 S_{F}^{X_{1}}+I_{H} .
\end{aligned}
$$

It follows that

$$
\left(S_{F}^{X_{1}}\right)^{2}+\left(S_{F}^{X_{1}^{c}}\right)^{2} \geq \frac{1}{2} I_{H} .
$$

by Lemma 1 . Also observing that $S_{F}^{X_{1}}-\left(S_{F}^{X_{1}}\right)^{2} \geq 0$ and

$$
\begin{aligned}
\left(S_{F}^{X_{1}}\right)^{2}+\left(S_{F}^{X_{1}^{c}}\right)^{2} & =2\left(S_{F}^{X_{1}}\right)^{2}-2 S_{F}^{X_{1}}+I_{H} \\
& =I_{H}+2 S_{F}^{X_{1}}-2\left(S_{F}^{X_{1}}\right)^{2}+4\left(\left(S_{F}^{X_{1}}\right)^{2}-S_{F}^{X_{1}}\right),
\end{aligned}
$$

we have

$$
\left(S_{F}^{X_{1}}\right)^{2}+\left(S_{F}^{X_{1}^{c}}\right)^{2} \leq I_{H}+2 S_{F}^{X_{1}}-2\left(S_{F}^{X_{1}}\right)^{2} .
$$

Again by Lemma 1, we get

$$
\left(S_{F}^{X_{1}}\right)^{2}+\left(S_{F}^{X_{1}^{c}}\right)^{2} \leq \frac{3}{2} I_{H}
$$

Since

$$
\begin{aligned}
\left\langle\left(S_{F}^{X_{1}}\right)^{2}+\left(S_{F}^{X_{1}^{c}}\right)^{2} h, h\right\rangle & =\left\langle S_{F}^{X_{1}} h, S_{F}^{X_{1}} h\right\rangle+\left\langle S_{F}^{X_{1}^{c}} h, S_{F}^{X_{1}^{c}} h\right\rangle \\
& =\left\|\int_{X_{1}} v^{2}(x) \pi_{F(x)}(h) d \mu(x)\right\|^{2}+\left\|\int_{X_{1}^{c}} v^{2}(x) \pi_{F(x)}(h) d \mu(x)\right\|^{2}
\end{aligned}
$$

for $h \in H$, we have (7) by (13) and (15).

Next we prove (8). Observe that

$$
\begin{aligned}
S_{F}^{X_{1}}+\left(S_{F}^{X_{1}^{c}}\right)^{2} & =S_{F}^{X_{1}}+\left(I_{H}-S_{F}^{X_{1}}\right)^{2} \\
& =\left(S_{F}^{X_{1}}\right)^{2}-S_{F}^{X_{1}}+I_{H},
\end{aligned}
$$


and that $S_{F}^{X_{1}}-\left(S_{F}^{X_{1}}\right)^{2} \geq 0$ by (9). It leads to

$$
\frac{3}{4} I_{H} \leq S_{F}^{X_{1}}+\left(S_{F}^{X_{1}^{c}}\right)^{2} \leq I_{H}
$$

by Lemma 1. For $h \in H$, we have

$$
\begin{aligned}
\left\langle S_{F}^{X_{1}}+\left(S_{F}^{X_{1}^{c}}\right)^{2} h, h\right\rangle & =\left\langle S_{F}^{X_{1}} h, h\right\rangle+\left\langle S_{F}^{X_{1}^{c}} h, S_{F}^{X_{1}^{c}} h\right\rangle \\
& =\int_{X_{1}} v^{2}(x)\left\|\pi_{F(x)}(h)\right\|^{2} d \mu(x)+\left\|\int_{X_{1}^{c}} v^{2}(x) \pi_{F(x)}(h) d \mu(x)\right\|^{2} .
\end{aligned}
$$

Collecting (18) and (19) leads to (8). The proof is completed.

Observe that $\left(\frac{1}{\sqrt{\lambda}} F, v\right)$ is a Parseval continuous fusion frame if $(F, v)$ is a $\lambda$-tight continuous fusion frame for $H$. As an immediate consequence of Theorem 3.1, we have

Corollary 1 Let $(F, v)$ be a $\lambda$-tight continuous fusion frame for $H$. Then for $X_{1} \subset X$ and $h \in H$, we have

$$
\begin{aligned}
0 & \leq \lambda \int_{X_{1}} v^{2}(x)\left\|\pi_{F(x)}(h)\right\|^{2} d \mu(x)-\left\|\int_{X_{1}} v^{2}(x) \pi_{F(x)}(h) d \mu(x)\right\|^{2} \leq \frac{\lambda^{2}}{4}\|h\|^{2} \\
\frac{\lambda^{2}}{2}\|h\|^{2} & \leq\left\|\int_{X_{1}} v^{2}(x) \pi_{F(x)}(h) d \mu(x)\right\|^{2}+\left\|\int_{X_{1}^{c}} v^{2}(x) \pi_{F(x)}(h) d \mu(x)\right\|^{2} \leq \frac{3 \lambda^{2}}{2}\|h\|^{2} \\
\frac{3 \lambda^{2}}{4}\|h\|^{2} & \leq \lambda \int_{X_{1}} v^{2}(x)\left\|\pi_{F(x)}(h)\right\|^{2} d \mu(x)+\left\|\int_{X_{1}^{c}} v^{2}(x) \pi_{F(x)}(h) d \mu(x)\right\|^{2} \leq \lambda^{2}\|h\|^{2} .
\end{aligned}
$$

Next we will give a equality for tight continuous fusion frames. To do so, we first define two operators $S_{F}^{1}$, $S_{F}^{2}$ as follows:

$$
\begin{aligned}
& S_{F}^{1}: H \rightarrow H, \quad S_{F}^{1}(h)=\int_{X} a_{x} v^{2}(x) \pi_{F(x)}(h) d \mu(x), \quad \forall h \in H, \\
& S_{F}^{2}: H \rightarrow H, \quad S_{F}^{2}(h)=\int_{X}\left(1-a_{x}\right) v^{2}(x) \pi_{F(x)}(h) d \mu(x), \quad \forall h \in H,
\end{aligned}
$$

where $(F, v)$ is a continuous Bessel fusion mapping on $H$ and $\left\{a_{x}: x \in X\right\} \in l^{\infty}(X)$, $l^{\infty}(X)=\left\{\left\{a_{x}: x \in X\right\}: \sup _{x \in X}\left|a_{x}\right|<\infty\right\}$.

Proposition 4 Let $(F, v)$ be a continuous Bessel fusion mapping on $H$ with bound B, then $S_{F}^{1}$, $S_{F}^{2}$ are bounded linear operators, and

$$
\begin{aligned}
& \left(S_{F}^{1}\right)^{*}(h)=\int_{X} \bar{a}_{x} v^{2}(x) \pi_{F(x)}(h) d \mu(x), \quad \forall h \in H . \\
& \left(S_{F}^{2}\right)^{*}(h)=\int_{X}\left(1-\bar{a}_{x}\right) v^{2}(x) \pi_{F(x)}(h) d \mu(x), \quad \forall h \in H .
\end{aligned}
$$


Proof We only treat $S_{F}^{1}$, and the other part $S_{F}^{2}$ can be treated similarly. For $h \in H$ and $X_{1} \subset X$, we have

$$
\begin{aligned}
\left\|\int_{X_{1}} a_{x} v^{2}(x) \pi_{F(x)}(h) d \mu(x)\right\| & =\sup _{g \in H,\|g\|=1}\left|\left\langle\int_{X_{1}} a_{x} v^{2}(x) \pi_{F(x)}(h) d \mu(x), g\right\rangle\right| \\
= & \sup _{g \in H,\|g\|=1}\left|\int_{X_{1}} v^{2}(x)\left\langle\pi_{F(x)} h, \bar{a}_{x} g\right\rangle d \mu(x)\right| \\
= & \sup _{g \in H,\|g\|=1}\left|\int_{X_{1}} v^{2}(x)\left\langle\pi_{F(x)} h, \bar{a}_{x} \pi_{F(x)} g\right\rangle d \mu(x)\right| \\
\leq & \sup _{g \in H,\|g\|=1}\left(\int_{X_{1}} v^{2}(x)\left\|\pi_{F(x)}(h)\right\|^{2} d \mu(x)\right)^{\frac{1}{2}} \\
& \times\left(\int_{X_{1}} v^{2}(x)\left\|\bar{a}_{x} \pi_{F(x)}(h)\right\|^{2} d \mu(x)\right)^{\frac{1}{2}} \\
\leq & B M\|h\|,
\end{aligned}
$$

where $M=\sup _{x \in X}\left|a_{x}\right|$ and $\bar{a}_{x}$ is the conjugate of $a_{x}$. This implies that $S_{F}^{1}$ is well-defined and $\left\|S_{F}^{1} h\right\| \leq B M\|h\|$. Therefore, $S_{F}^{1}$ is a bounded linear operator. Now let us compute $\left(S_{F}^{1}\right)^{*}$,

$$
\begin{aligned}
\left\langle h,\left(S_{F}^{1}\right)^{*}(f)\right\rangle=\left\langle S_{F}^{1} h, f\right\rangle & =\left\langle\int_{X} a_{x} v^{2}(x) \pi_{F(x)}(h) d \mu(x), f\right\rangle \\
& =\int_{X} v^{2}(x)\left\langle\pi_{F(x)}(h), \bar{a}_{x} f\right\rangle d \mu(x) \\
& =\int_{X} v^{2}(x)\left\langle h, \bar{a}_{x} \pi_{F(x)}(f)\right\rangle d \mu(x) \\
& =\left\langle h, \int_{X} \bar{a}_{x} v^{2}(x) \pi_{F(x)}(f) d \mu(x)\right\rangle .
\end{aligned}
$$

The proof is completed.

Theorem 2 Let $(F, v)$ be a $\lambda$-tight continuous fusion frame for $H$. Then for $h \in H$ and $\left\{a_{x}: x \in X\right\} \in l^{\infty}(X)$, we have

$$
\begin{aligned}
& \lambda \int_{X} a_{x} v^{2}(x)\left\|\pi_{F(x)}(h)\right\|^{2} d \mu(x)+\left\|\int_{X}\left(1-a_{x}\right) v^{2}(x) \pi_{F(x)}(h) d \mu(x)\right\|^{2} \\
& \quad=\lambda \int_{X}\left(1-\bar{a}_{x}\right) v^{2}(x)\left\|\pi_{F(x)}(h)\right\|^{2} d \mu(x)+\left\|\int_{X} a_{x} v^{2}(x) \pi_{F(x)}(h) d \mu(x)\right\|^{2},
\end{aligned}
$$

where $\bar{a}_{x}$ is the conjugate of $a_{x}$.

Proof By Proposition 4, $S_{F}^{1}, S_{F}^{2}$ are well-defined and

$$
S_{F}^{1} h+S_{F}^{2} h=\int_{X} v^{2}(x) \pi_{F(x)}(h) d \mu(x)=S_{F} h, \quad \forall h \in H .
$$


Since $(F, v)$ is a $\lambda$-tight continuous fusion frame for $H$, that is $\lambda^{-1} S_{F}^{1}+\lambda^{-1} S_{F}^{2}=I_{H}$. Write $Q_{1}=\lambda^{-1} S_{F}^{1}$ and $Q_{2}=\lambda^{-1} S_{F}^{2}$, then

$$
\begin{aligned}
Q_{1}+Q_{2}^{*} Q_{2} & =Q_{1}+\left(I_{H}-Q_{1}\right)^{*}\left(I_{H}-Q_{1}\right) \\
& =Q_{1}+\left(I_{H}-Q_{1}^{*}\right)\left(I_{H}-Q_{1}\right) \\
& =Q_{1}+I_{H}-Q_{1}-Q_{1}^{*}+Q_{1}^{*} Q_{1} \\
& =I_{H}-Q_{1}^{*}+Q_{1}^{*} Q_{1} \\
& =Q_{2}^{*}+Q_{1}^{*} Q_{1},
\end{aligned}
$$

and thus

$$
\lambda S_{F}^{1}+\left(S_{F}^{2}\right)^{*} S_{F}^{2}=\lambda S_{F}^{2}+\left(S_{F}^{1}\right)^{*} S_{F}^{1} .
$$

Hence for $h \in H$, we get

$$
\begin{aligned}
& \lambda \int_{X} a_{x} v^{2}(x)\left\|\pi_{F(x)}(h)\right\|^{2} d \mu(x)+\left\|\int_{X}\left(1-a_{x}\right) v^{2}(x) \pi_{F(x)}(h) d \mu(x)\right\|^{2} \\
& \quad=\left\langle\lambda S_{F}^{1} h, h\right\rangle+\left\langle\left(S_{F}^{2}\right)^{*} S_{F}^{2} h, h\right\rangle \\
& \quad=\left\langle\left(\lambda S_{F}^{1}+\left(S_{F}^{2}\right)^{*} S_{F}^{2}\right) h, h\right\rangle \\
& \quad=\left\langle\left(\lambda S_{F}^{2}+\left(S_{F}^{1}\right)^{*} S_{F}^{1}\right) h, h\right\rangle \\
& =\left\langle\lambda\left(S_{F}^{2}\right)^{*} h, h\right\rangle+\left\langle\left(S_{F}^{1}\right)^{*} S_{F}^{1} h, h\right\rangle \\
& \quad=\left\langle h, S_{F}^{2} h\right\rangle+\left\|S_{F}^{1} h\right\|^{2} \\
& \quad=\lambda \int_{X}\left(1-\bar{a}_{x}\right) v^{2}(x)\left\|\pi_{F(x)}(h)\right\|^{2} d \mu(x)+\left\|\int_{X} a_{x} v^{2}(x) \pi_{F(x)}(h) d \mu(x)\right\|^{2} .
\end{aligned}
$$

The proof is completed.

As an immediate consequence of Theorem 2 and Lemma 2, we have

Corollary 2 Let $(F, v)$ be a $\lambda$-tight continuous fusion frame for $H,\left\{a_{x}: x \in X\right\} \in l^{\infty}(X)$ with $a_{x}$ being real. Then for $h \in H$, we have

$$
\begin{aligned}
& \lambda \int_{X} a_{x} v^{2}(x)\left\|\pi_{F(x)}(h)\right\|^{2} d \mu(x)+\left\|\int_{X}\left(1-a_{x}\right) v^{2}(x) \pi_{F(x)}(h) d \mu(x)\right\|^{2} \\
& \quad=\lambda \int_{X}\left(1-a_{x}\right) v^{2}(x)\left\|\pi_{F(x)}(h)\right\|^{2} d \mu(x)+\left\|\int_{X} a_{x} v^{2}(x) \pi_{F(x)}(h) d \mu(x)\right\|^{2} \geq \frac{3}{4}\|h\|^{2} .
\end{aligned}
$$

\section{Equalities and inequalities for fusion pairs}

This section focuses on fusion pairs. We begin with the following lemma which can be proved similarly to Proposition 4.

Lemma 3 Let $(F, v)$ and $(G, v)$ be continuous Bessel fusion mappings on $H, F$ and $G$ be a fusion pair, and $\left\{a_{x}: x \in X\right\} \in l^{\infty}(X)$. Define the operator $T_{a}$ as follows:

$$
T_{a}: H \rightarrow H, T_{a} h=\int_{X} a_{x} v^{2}(x) \pi_{F(x)} \pi_{G(x)}(h) d \mu(x), \quad \forall h \in H ;
$$


then $T_{a}$ is a bounded linear operator, and

$$
T_{a}^{*} h=\int_{X} \bar{a}_{x} v^{2}(x) \pi_{G(x)} \pi_{F(x)}(h) d \mu(x)
$$

Theorem 3 Let $(F, v)$ and $(G, v)$ be continuous Bessel fusion mappings on $H, F$ and $G$ be a fusion pair, and $\left\{a_{x}: x \in X\right\} \in l^{\infty}(X)$. Then for $h \in H$, we have

$$
\begin{aligned}
& \left\|\int_{X} a_{x} v^{2}(x) \pi_{F(x)} \pi_{G(x)}(h) d \mu(x)\right\|^{2}+\int_{X}\left(1-a_{x}\right) v^{2}(x)\left\langle\pi_{G(x)}(h), \pi_{F(x)}(h)\right\rangle d \mu(x) \\
& =\left\|\int_{X}\left(1-a_{x}\right) v^{2}(x) \pi_{F(x)} \pi_{G(x)}(h) d \mu(x)\right\|^{2}+\overline{\int_{X} a_{x} v^{2}(x)\left\langle\pi_{G(x)}(h), \pi_{F(x)}(h)\right\rangle d \mu(x)}
\end{aligned}
$$

Proof Let $T_{1-a} h=\int_{X}\left(1-a_{x}\right) v^{2}(x) \pi_{F(x)} \pi_{G(x)}(h) d \mu(x), \forall h \in H$, then

$$
T_{1-a}^{*} h=\int_{X}\left(1-\bar{a}_{x}\right) v^{2}(x) \pi_{G(x)} \pi_{F(x)}(h) d \mu(x), \quad \forall h \in H
$$

and $T_{a}+T_{1-a}=I_{H}$. So for $h \in H$, we have

$$
\begin{aligned}
& \left\|\int_{X} a_{x} v^{2}(x) \pi_{F(x)} \pi_{G(x)}(h) d \mu(x)\right\|^{2}+\int_{X}\left(1-a_{x}\right) v^{2}(x)\left\langle\pi_{G(x)}(h), \pi_{F(x)}(h)\right\rangle d \mu(x) \\
& \quad=\left\|T_{a} h\right\|^{2}+\left\langle T_{1-a} h, h\right\rangle=\left\langle T_{a} h, T_{a} h\right\rangle+\left\langle\left(I_{H}-T_{a}\right) h, h\right\rangle \\
& \quad=\left\langle T_{a} h, T_{a} h\right\rangle+\langle h, h\rangle-\left\langle T_{a} h, h\right\rangle .
\end{aligned}
$$

On the other hand,

$$
\begin{aligned}
& \left\|\int_{X}\left(1-a_{x}\right) v^{2}(x) \pi_{F(x)} \pi_{G(x)}(h) d \mu(x)\right\|^{2}+\overline{\int_{X} a_{x} v^{2}(x)\left\langle\pi_{G(x)}(h), \pi_{F(x)}(h)\right\rangle d \mu(x)} \\
& \quad=\left\|T_{1-a} h\right\|^{2}+\overline{\left\langle T_{a} h, h\right\rangle}=\left\langle T_{1-a} h, T_{1-a} h\right\rangle+\left\langle h, T_{a} h\right\rangle \\
& \quad=\left\langle\left(I_{H}-T_{a}\right) h,\left(I_{H}-T_{a}\right) h\right\rangle+\left\langle h, T_{a} h\right\rangle \\
& \quad=\langle h, h\rangle-\left\langle T_{a} h, h\right\rangle+\left\langle T_{a} h, T_{a} h\right\rangle .
\end{aligned}
$$

Therefore, (22) holds by (23) and (24). The proof is completed.

Remark 2 Theorem 2 is a special case of Theorem 3.

Theorem 4 Let $(F, v)$ and $(G, v)$ be continuous Bessel fusion mappings on $H, F$ and $G$ be a fusion pair, and $\left\{a_{x}: x \in X\right\} \in l^{\infty}(X)$. Then for $h \in H$, we have

$$
\begin{aligned}
& \left\|\int_{X} a_{x} v^{2}(x) \pi_{F(x)} \pi_{G(x)}(h) d \mu(x)\right\|^{2}+\operatorname{Re}\left(\int_{X}\left(1-a_{x}\right) v^{2}(x)\left\langle\pi_{G(x)}(h), \pi_{F(x)}(h)\right\rangle d \mu(x)\right) \\
& =\left\|\int_{X}\left(1-a_{x}\right) v^{2}(x) \pi_{F(x)} \pi_{G(x)}(h) d \mu(x)\right\|^{2} \\
& \quad+\operatorname{Re}\left(\int_{X} a_{x} v^{2}(x)\left\langle\pi_{G(x)}(h), \pi_{F(x)}(h)\right\rangle d \mu(x)\right) \geq \frac{3}{4}\|h\|^{2}
\end{aligned}
$$


Proof By Theorem 3, for $h \in H$, we have

$$
\begin{aligned}
& \left\|\int_{X} a_{x} v^{2}(x) \pi_{F(x)} \pi_{G(x)}(h) d \mu(x)\right\|^{2}+\operatorname{Re}\left(\int_{X}\left(1-a_{x}\right) v^{2}(x)\left\langle\pi_{G(x)}(h), \pi_{F(x)}(h)\right\rangle d \mu(x)\right) \\
& =\left\|\int_{X}\left(1-a_{x}\right) v^{2}(x) \pi_{G(x)} \pi_{F(x)}(h) d \mu(x)\right\|^{2}+\operatorname{Re}\left(\int_{X} a_{x} v^{2}(x)\left\langle\pi_{F(x)}(h), \pi_{G(x)}(h)\right\rangle d \mu(x)\right) .
\end{aligned}
$$

Next we prove the "inequality" part. By Lemma 3, we have

$$
T_{a} h=\int_{X} a_{x} v^{2}(x) \pi_{F(x)} \pi_{G(x)}(h) d \mu(x), \quad \forall h \in H,
$$

and

$$
T_{a}^{*} h=\int_{X} \bar{a}_{x} v^{2}(x) \pi_{G(x)} \pi_{F(x)}(h) d \mu(x), \quad \forall h \in H .
$$

It follows that

$$
\operatorname{Re}\left(\int_{X} a_{x} v^{2}(x)\left\langle\pi_{G(x)}(h), \pi_{F(x)}(h)\right\rangle d \mu(x)\right)=\left\langle\frac{T_{a}+T_{a}^{*}}{2} h, h\right\rangle .
$$

Thus for $h \in H$, we have

$$
\begin{aligned}
& \left\|\int_{X}\left(1-a_{x}\right) v^{2}(x) \pi_{F(x)} \pi_{G(x)}(h) d \mu(x)\right\|^{2}+R e\left(\int_{X} a_{x} v^{2}(x)\left\langle\pi_{G(x)}(h), \pi_{F(x)}(h)\right\rangle d \mu(x)\right) \\
& =\left\langle\left(T_{1-a}^{*} T_{1-a}+\frac{T_{a}+T_{a}^{*}}{2}\right) h, h\right\rangle \\
& =\left\langle\left(\left(I_{H}-T_{a}^{*}\right)\left(I_{H}-T_{a}\right)+\frac{T_{a}+T_{a}^{*}}{2}\right) h, h\right\rangle \\
& =\left\langle\left(I_{H}+T_{a}^{*} T_{a}-\frac{T_{a}+T_{a}^{*}}{2}\right) h, h\right\rangle \\
& =\left\langle\left[\left(T_{a}-\frac{1}{2} I_{H}\right)^{*}\left(T_{a}-\frac{1}{2} I_{H}\right)+\frac{3}{4} I\right] h, h\right\rangle \\
& =\left\|\left(T_{a}-\frac{1}{2} I_{H}\right) h\right\|^{2}+\frac{3}{4}\|h\|^{2} \geq \frac{3}{4}\|h\|^{2} .
\end{aligned}
$$

The proof is completed.

Remark 3 Corollary 2 is a special case of Theorem 4.

Take

$$
a_{x}=\left\{\begin{array}{rr}
1, & x \in X_{1} \\
0, & x \in X_{1}^{c}
\end{array}\right.
$$

in Theorem 3, where $X_{1} \subset X$. Then we have

Corollary 3 Let $X_{1} \subset X,(F, v)$ and $(G, v)$ be continuous Bessel fusion mappings on $H, F$ and $G$ be a fusion pair. Then for $h \in H$, we have 


$$
\begin{aligned}
& \left\|\int_{X_{1}} v^{2}(x) \pi_{F(x)} \pi_{G(x)}(h) d \mu(x)\right\|^{2}+\operatorname{Re}\left(\int_{X_{1}^{c}} v^{2}(x)\left\langle\pi_{G(x)}(h), \pi_{F(x)}(h)\right\rangle d \mu(x)\right) \\
& \quad=\left\|\int_{X_{1}^{c}} v^{2}(x) \pi_{F(x)} \pi_{G(x)}(h) d \mu(x)\right\|^{2}+\operatorname{Re}\left(\int_{X_{1}} v^{2}(x)\left\langle\pi_{G(x)}(h), \pi_{F(x)}(h)\right\rangle d \mu(x)\right) \geq \frac{3}{4}\|h\|^{2}
\end{aligned}
$$

Theorem 5 Let $(F, v)$ and $(G, v)$ be continuous Bessel fusion mappings on $H, F$ and $G$ be a fusion pair, and $\left\{a_{x}: x \in X\right\} \in l^{\infty}(X)$. Then for $h \in H$, we have

$$
\begin{aligned}
\frac{3}{4}\|h\|^{2} & \leq\left\|\int_{X} a_{x} v^{2}(x) \pi_{F(x)} \pi_{G(x)}(h) d \mu(x)\right\|^{2}+\operatorname{Re}\left(\int_{X}\left(1-a_{x}\right) v^{2}(x)\left\langle\pi_{G(x)}(h), \pi_{F(x)}(h)\right\rangle d \mu(x)\right) \\
& =\left\|\int_{X}\left(1-a_{x}\right) v^{2}(x) \pi_{F(x)} \pi_{G(x)}(h) d \mu(x)\right\|^{2}+\operatorname{Re}\left(\int_{X} a_{x} v^{2}(x)\left\langle\pi_{G(x)}(h), \pi_{F(x)}(h)\right\rangle d \mu(x)\right) \\
& \leq \frac{3+\left\|T_{a}-T_{1-a}\right\|^{2}}{4}\|h\|^{2}
\end{aligned}
$$

Proof By Theorem 4, the left-hand inequality of (27) holds. Next we prove the righthand inequality. Observe that $T_{a}+T_{1-a}=I_{H}$. For $h \in H$, we have

$$
\begin{aligned}
& \left\|\int_{X}\left(1-a_{x}\right) v^{2}(x) \pi_{F(x)} \pi_{G(x)}(h) d \mu(x)\right\|^{2}+\operatorname{Re}\left(\int_{X} a_{x} v^{2}(x)\left\langle\pi_{G(x)}(h), \pi_{F(x)}(h)\right\rangle d \mu(x)\right) \\
& \quad=\left\langle T_{1-a} h, T_{1-a} h\right\rangle+\operatorname{Re}\left\langle T_{a} h, h\right\rangle \\
& \quad=\left\langle T_{1-a} h, T_{1-a} h\right\rangle+\langle h, h\rangle-\operatorname{Re}\left\langle T_{1-a} h, h\right\rangle \\
& \quad=\frac{3}{4}\langle h, h\rangle+\frac{1}{4}\langle h, h\rangle-\operatorname{Re}\left\langle T_{1-a} h, h\right\rangle+\left\langle T_{1-a} h, T_{1-a} h\right\rangle \\
& \quad=\frac{3}{4}\langle h, h\rangle+\frac{1}{4}\left(\langle h, h\rangle-4 \operatorname{Re}\left\langle T_{1-a} h, h\right\rangle+4\left\langle T_{1-a} h, T_{1-a} h\right\rangle\right) \\
& \quad=\frac{3}{4}\langle h, h\rangle+\frac{1}{4}\left(\langle h, h\rangle-2\left\langle T_{1-a} h, h\right\rangle-2\left\langle h, T_{1-a} h\right\rangle+4\left\langle T_{1-a} h, T_{1-a} h\right\rangle\right) \\
& \quad=\frac{3}{4}\langle h, h\rangle+\frac{1}{4}\left\langle\left(I_{H}-2 T_{1-a}\right) h,\left(I_{H}-2 T_{1-a}\right) h\right\rangle \\
& \quad=\frac{3}{4}\langle h, h\rangle+\frac{1}{4}\left\langle\left(T_{a}-T_{1-a}\right) h,\left(T_{a}-T_{1-a}\right) h\right\rangle \\
& \quad \leq \frac{3}{4}\|h\|^{2}+\frac{1}{4}\left\|T_{a}-T_{1-a}\right\|^{2}\|h\|^{2} \\
& \quad=\frac{3+\left\|T_{a}-T_{1-a}\right\|^{2}\|h\|^{2} .}{4}
\end{aligned}
$$

The proof is completed.

Let $X_{1} \subset X,(F, v)$ and $(G, v)$ be continuous Bessel fusion mappings on $H, F$ and $G$ be a fusion pair. Define the operators $L_{X_{1}}, L_{X_{1}^{c}}$ as follows:

$$
\begin{aligned}
& L_{X_{1}}: H \rightarrow H, L_{X_{1}} h=\int_{X_{1}} v^{2}(x) \pi_{F(x)} \pi_{G(x)}(h) d \mu(x), \quad \forall h \in H ; \\
& L_{X_{1}^{c}}: H \rightarrow H, L_{X_{1}^{c}} h=\int_{X_{1}^{c}} v^{2}(x) \pi_{F(x)} \pi_{G(x)}(h) d \mu(x), \quad \forall h \in H .
\end{aligned}
$$

It is easy to prove $L_{X_{1}}, L_{X_{1}^{c}}$ are bounded linear operators. As an immediate consequence of Theorem 5 and Corollary 3 , we have 
Corollary 4 Let $X_{1} \subset X,(F, v)$ and $(G, v)$ be continuous Bessel fusion mappings for $H, F$ and $G$ be a fusion pair. Then for $h \in H$, we have

$$
\begin{aligned}
\frac{3}{4}\|h\|^{2} & \leq\left\|\int_{X_{1}} v^{2}(x) \pi_{F(x)} \pi_{G(x)}(h) d \mu(x)\right\|^{2}+\operatorname{Re}\left(\int_{X_{1}^{c}} v^{2}(x)\left\langle\pi_{G(x)}(h), \pi_{F(x)}(h)\right\rangle d \mu(x)\right) \\
& =\left\|\int_{X_{1}^{c}} v^{2}(x) \pi_{F(x)} \pi_{G(x)}(h) d \mu(x)\right\|^{2}+\operatorname{Re}\left(\int_{X_{1}} v^{2}(x)\left\langle\pi_{G(x)}(h), \pi_{F(x)}(h)\right\rangle d \mu(x)\right) \\
& \leq \frac{3+\left\|L_{X_{1}}-L_{X_{1}^{c}}\right\|^{2}}{4}\|h\|^{2}
\end{aligned}
$$

\section{Conclusions}

In this paper, we obtain three inequalities for Parseval continuous fusion frames, an equality for $\lambda$-tight continuous fusion frames and an inequality for fusion pairs (see Theorems 3.1, 3.2 and 4.3). These results can recover some well known frames inequalities.

\section{Authors' contributions}

This work was carried out in collaboration among the authors. All authors have a good contribution to design the study, and to perform the analysis of this research work. All authors read and approved the final manuscript.

\section{Acknowledgements}

The authors would like to thank the referees for carefully reviewing this manuscript and for providing valuable comments, which greatly improve its quality. Supported by the National Natural Science Foundation of China (Grant No. 11271037).

\section{Competing interests}

The authors declare that they have no competing interests.

Received: 14 May 2016 Accepted: 11 September 2016

Published online: 19 September 2016

\section{References}

Balan R, Casazza PG, Edidin D (2006) Signal reconstruction without noisy phase. Appl Comput Harmon Anal 20:345-356 Balan R, Casazza PG, Edidin D, Kutyniok G (2005) Decompositions of frames and a new frame identity, vol 5914. In: SPIE proceedings of Wavelets XI, San Diego, CA, 2005. SPIE, Bellingham, pp 379-388

Balan R, Casazza PG, Edidin D, Kutyniok G (2007) A new identity for Parseval frames. Proc Am Math Soc 135:1007-1015 Bownik M, Luoto K, Richmond E (2015) A combinatorial characterization of tight fusion frames. Pac J Math 275:257-294 Casazza PG (2000) The art of frame theory. Taiwan J Math 4:129-201 Casazza PG, Kutyniok G (2004) Frame of subspace. Contemp Math 345:87-114

Casazza PG, Kutyniok G, Li S (2008) Fusion frames and distributed processing. Appl Comput Harmon Anal 25(1):114-132 Christensen $O$ (2003) An introduction to frame and Riesz base. Birkhäuser, Boston

Daubechies I, Grossmann A, Meyer Y (1986) Painess nonorthogonal expansion. J Math Phys 27:1271-1283

Duffin RJ, Schaeffer AC (1952) A class of nonharmonic Fourier series. Trans Am Soc 72:341-366

Faroughi MH, Ahmadi R (2008) C-Fusion frame. J Appl Sci 8(16):2881-2887

Faroughi MH, Ahmadi R (2010) Some properties of C-fusion frames. Turk J Math 34:393-415

Găvruţa P (2006) On some identities and inequalities for frames in Hilbert spaces. J Math Anal Appl 1:469-478

Guo Q, Leng J, Li H (2016) Some equalities and inequalities for fusion frames. SpringerPlus 5:121

Leng JS, Han DG (2013) Orthogonal projection decomposition of matrices and construction of fusion frames. Adv Comput Math 38:369-381

Li DF, Sun WC (2008) Some equalities and inequalities for generalizaed frames. Chin J Comtemp Math 29(3):301-308

Li J, Zhu Y (2012) Some equalities and inequalities for g-Bessel sequences in Hilbert spaces. Appl Math Lett 25(11):1601-1607

Li XB, Yang SZ, Zhu YC (2015) Some results about operator perturbation of fusion frames in Hilbert spaces. J Math Anal Appl 421:1417-1427

Rahimi A, Najati A, Dehghan YN (2006) Continuous frame in Hilbert space. Methods Funct Anal Topol 12:170-182

Strohmer T, Heath RW (2003) Grassmannian frames with applications to coding and communication. Appl Comput Harmon Anal 14:257-275

Zhu X, Wu G (2010) A note on some equalities for frames in Hilbert spaces. Appl Math Lett 23(7):788-790 